(c) 2016 IEEE. Personal use of this material is permitted. Permission from IEEE must be obtained for all other uses, in any current or future media, including reprinting/republishing this material for advertising or promotional purposes, creating new collective works, for resale or redistribution to servers or lists, or reuse of any copyrighted component of this work in other works. 


\title{
A semidefinite programming upper bound of quantum capacity
}

\author{
Xin Wang*, Runyao Duan*† \\ ${ }^{*}$ Centre for Quantum Computation and Intelligent Systems \\ Faculty of Engineering and Information Technology \\ University of Technology Sydney (UTS), NSW 2007, Australia \\ †UTS-AMSS Joint Research Laboratory for Quantum Computation and Quantum Information Processing \\ Academy of Mathematics and Systems Science \\ Chinese Academy of Sciences, Beijing 100190, China \\ Email: xin.wang-8@student.uts.edu.au,runyao.duan@uts.edu.au
}

\begin{abstract}
Recently the power of positive partial transpose preserving (PPTp) and no-signalling (NS) codes in quantum communication has been studied. We continue with this line of research and show that the NS/PPTp/NS $\cap$ PPTp codes assisted zero-error quantum capacity depends only on the noncommutative bipartite graph of the channel and the one-shot case can be computed efficiently by semidefinite programming (SDP). As an example, the activated PPTp codes assisted zeroerror quantum capacity is carefully studied. We then present a general SDP upper bound $Q_{\Gamma}$ of quantum capacity and show it is always smaller than or equal to the "Partial transposition bound" introduced by Holevo and Werner, and the inequality could be strict. This upper bound is found to be additive, and thus is an upper bound of the potential PPTp assisted quantum capacity as well. We further demonstrate that $Q_{\Gamma}$ is strictly better than several previously known upper bounds for an explicit class of quantum channels. Finally, we show that $Q_{\Gamma}$ can be used to bound the super-activation of quantum capacity.
\end{abstract}

\section{INTRODUCTION}

A fundamental problem in quantum information theory is to determine the quantum capacity of quantum channels. The quantum capacity of a noisy quantum channel is the highest rate at which it can convey quantum information reliably over asymptotically many uses of the channel. Quantum capacity is complicated to evaluate since it is characterized by a multiletter, regularized expression, and it is not even known to be computable [1]. Even for the low dimensional channels such as the qubit depolarizing channel, the quantum capacity remains unknown.

To deal with the intractable problem of determining quantum capacities of channels, assistance such as entanglement or classical communication have been introduced into the capacity problem [2], [3]. Particularly, positive partial transpose preserving (PPTp) and no-signalling (NS) codes assisted quantum capacity has been studied [3], which regards a channel code as a bipartite operation with an encoder belonging to the sender and a decoder belonging to the receiver.

Given an arbitrary quantum channel, the only known general computable upper bound is the partial transposition bound introduced in [4]. Other known upper bounds [5], [6], [7], [8], [9], [10], [11], [12] all require specific settings to be tight and computable. For example, the upper bound from no cloning argument [8], [9] only behaves well at very high noise levels. Also, upper bound raised by approximate degradable quantum channels [6] can evaluate the quantum capacity of arbitrary channels based on the single-letter capacity and this usually works well just for approximate degradable quantum channels. Thus it is of great interest and significance to find an efficiently computable upper bound for quantum capacity.

Before we present our main results, let us first review some notations and preliminaries. Let $\mathcal{N}(\rho)=\sum_{k} E_{k} \rho E_{k}^{\dagger}$ be a quantum channel from $\mathcal{L}\left(A^{\prime}\right)$ to $\mathcal{L}(B)$, where $\sum_{k} E_{k}^{\dagger} E_{k}=$ $\mathbb{1}_{A^{\prime}}$. The Choi-Jamiołkowski matrix of $\mathcal{N}$ is given by $J_{A B}=$ $\sum_{i j}|i\rangle\left\langle\left. j\right|_{A} \otimes \mathcal{N}\left(|i\rangle\left\langle\left. j\right|_{A^{\prime}}\right)=\left(\operatorname{id}_{A} \otimes \mathcal{N}\right)\left|\Phi_{A A^{\prime}}\right\rangle\left\langle\Phi_{A A^{\prime}}\right|\right.\right.$, where $A$ and $A^{\prime}$ are isomorphic Hilbert spaces with respective orthonormal basis $\{|i\rangle\}$ and $\{|j\rangle\}$, and $\left|\Phi_{A A^{\prime}}\right\rangle=\sum_{k}\left|k_{A}\right\rangle\left|k_{A^{\prime}}\right\rangle$ is the unnormalized maximally-entangled state over $A \otimes A^{\prime}$. And $K=K(\mathcal{N})=\operatorname{span}\left\{E_{k}\right\}$ denotes the Choi-Kraus operator space of $\mathcal{N}$. The coherent information of $\mathcal{N}$ is given by

$$
\mathrm{I}_{\mathrm{C}}(\mathcal{N})=\max _{\rho_{A}} \mathrm{H}\left(\mathcal{N}\left(\rho_{A}\right)\right)-\mathrm{H}\left(\mathcal{N}^{c}\left(\rho_{A}\right)\right),
$$

where $\mathcal{N}^{c}$ is the complementary channel of $\mathcal{N}$ and $\mathrm{H}(\sigma)=$ $-\operatorname{Tr}(\sigma \log \sigma)$ denotes the von Neumann entropy of a density operator $\sigma$. The work in [13], [14], [15] showed that coherent information of $\mathcal{N}$ is an achievable rate for quantum communication while the work in [16], [17], [18] showed the regularized coherent information is also an upper bound on quantum capacity. This establishes that

$$
\mathrm{Q}(\mathcal{N})=\lim _{n \rightarrow \infty} \frac{\mathrm{I}_{\mathrm{C}}\left(\mathcal{N}^{\otimes n}\right)}{n} .
$$

A general "code" is defined as a set of operations performed by the sender Alice and the receiver Bob which can be used to improve the data transmission with the given channel [3]. The PPTp codes are those for which the bipartite operation is PPT-preserving. A nonzero positive semi-definite operator $E \in$ $\mathcal{L}(\mathcal{X} \otimes \mathcal{Y})$ is said to be a positive partial transpose operator (or simply PPT) if $E^{T_{\mathcal{X}}} \geq 0$, where $T_{\mathcal{X}}$ means the partial transpose with respect to the party $\mathcal{X}$, i.e., $(|i j\rangle\langle k l|)^{T_{\mathcal{X}}}=|k j\rangle\langle i l|$. A bipartite operation $\Pi: \mathcal{L}\left(A_{i} \otimes B_{i}\right) \rightarrow \mathcal{L}\left(A_{o} \otimes B_{o}\right)$ is 'PPTpreserving' if it sends any state which is PPT with respect to the Alice/Bob partition to another PPT state. As shown in [19], 
a bipartite operation $\Pi^{A_{i} \otimes B_{i} \rightarrow A_{o} \otimes B_{o}}$ is PPT-preserving if and only if its Choi-Jamiołkowski matrix $Z_{A_{i} B_{i} A_{o} B_{o}}$ is PPT.

The PPT-preserving operations include all operations that can be implemented by local operations and classical communication (LOCC) and were introduced to study entanglement distillation in an early paper by Rains [19]. They also include all unassisted and forward-classical-assisted codes introduced in [3]. The no-signalling (NS) codes refer to the bipartite quantum operations with the no-signalling constraints and this kind of codes are also useful in classical zero-error communication [20], [21], [22]. Let $\Omega$ represent NS, PPTp or NS $\cap$ PPTp in the rest of the paper. Given a channel $\mathcal{N}: \mathcal{L}(A) \rightarrow \mathcal{L}(B)$ and the $\Omega$ code of size $k$, the optimal channel fidelity is given by the following SDP [3]:

$$
\begin{gathered}
F^{\Omega}(\mathcal{N}, k)=\max \operatorname{Tr} J_{A B} W_{A B} \\
\text { s.t. } 0 \leq W_{A B} \leq \rho_{A} \otimes \mathbb{1}_{B}, \operatorname{Tr} \rho_{A}=1, \\
\text { PPTp: }-\frac{\rho_{A} \otimes \mathbb{1}_{B}}{k} \leq W_{A B}^{T_{B}} \leq \frac{\rho_{A} \otimes \mathbb{1}_{B}}{k}, \\
\text { NS: } \operatorname{Tr}_{A} W_{A B}=\frac{1}{k^{2}} \mathbb{1}_{B} .
\end{gathered}
$$

And the dual SDP is given by

$$
\begin{aligned}
F_{d}^{\Omega}(\mathcal{N}, k) & =\min \mu+k^{-2} \operatorname{Tr} S_{B} \\
\text { s.t. } & J_{A B}+\left(Y_{A B}-V_{A B}\right)^{T_{B}} \leq X_{A B}+\mathbb{1}_{A} \otimes S_{B}, \\
& \operatorname{Tr}_{B}\left(X_{A B}+k^{-1}\left(Y_{A B}+V_{A B}\right) \leq \mu \mathbb{1}_{A},\right. \\
& X_{A B}, Y_{A B}, V_{A B} \geq 0 .
\end{aligned}
$$

To remove the PPTp constraint, set $Y_{A B}=V_{A B}=0$. To remove the NS constraint, set $S_{B}=0$. The strong duality holds for $F^{P P T p}(\mathcal{N}, k)$, then $F^{P P T p}(\mathcal{N}, k)=F_{d}^{P P T p}(\mathcal{N}, k)$. Leung and Matthews [3] further introduced the quantum data transmission via quantum channels assisted with $\Omega$ codes. The $\Omega$ codes assisted zero-error quantum capacity is given by

$Q_{0}^{\Omega}(\mathcal{N})=\sup _{n} \max \left\{\frac{1}{n} \log k_{n}: F^{\Omega}\left(\mathcal{N}^{\otimes n}, k_{n}\right)=1, k_{n} \geq 0\right\}$.

When $n=1, Q_{0}^{\Omega,(1)}(\mathcal{N})=\left\lfloor\kappa^{\Omega}(\mathcal{N})\right\rfloor$ is the one-shot $\Omega$ codes assisted zero-error quantum capacity, where

$$
\kappa^{\Omega}(\mathcal{N}):=\max \left\{k: F^{\Omega}(\mathcal{N}, k)=1, k \geq 0\right\},
$$

and $\left\lfloor\kappa^{\Omega}(\mathcal{N})\right\rfloor$ means the integer part of $\kappa^{\Omega}(\mathcal{N})$. The corresponding quantum capacity is given by

$$
Q^{\Omega}(\mathcal{N}):=\sup \left\{r: \lim _{n \rightarrow \infty}: F^{\Omega}\left(\mathcal{N}^{\otimes n},\left\lfloor 2^{r n}\right\rfloor\right)=1\right\} .
$$

The so-called "non-commutative graph theory" was first suggested in [23]. The non-commutative graph associated with the channel captures the zero-error communication properties, thus playing a similar role to confusability graph of a classical channel. The zero-error classical capacity of a quantum channel in the presence of quantum feedback only depends on the Choi-Kraus operator space of the channel [24]. That is to say, the Choi-Kraus operator space $K$ plays a role that is quite similar to the bipartite graph and $K$ is alternatively called "non-commutative bipartite graph" [21]. Based on the idea in [25], we also define the potential $\Omega$ codes assisted quantum capacity

$$
Q_{p}^{\Omega}(\mathcal{N}):=\sup _{\mathcal{M}}\left[Q_{p}^{\Omega}(\mathcal{N} \otimes \mathcal{M})-Q_{p}^{\Omega}(\mathcal{M})\right] .
$$

In this paper, we first connect $\Omega$ codes assisted zero-error quantum capacity to the non-commutative bipartite graph. We then introduce the activated PPTp codes assisted zero-error quantum capacity. Furthermore, we present a general SDP upper bound $Q_{\Gamma}$ of quantum capacity. A general upper bound is usually difficult to find, however, our upper bound $Q_{\Gamma}$ can be applied to evaluate the quantum capacity of an arbitrary channel efficiently, whereas most previous upper bounds rely on specific conditions which can be different for each channel. We show that $Q_{\Gamma}$ is always smaller than or equal to the "Partial transposition bound" and the inequality can be strict. $Q_{\Gamma}$ is additive under tensor product, and thus is an upper bound of the potential PPTp assisted quantum capacity. We also demonstrate that this SDP upper bound is strictly better than several known upper bounds by explicit examples. For the super-activation of quantum capacity [26], $Q_{\Gamma}$ can also be applied to evaluate the super-activation.

\section{ASSISTED ZERO-ERROR QUANTUM CAPACITY AND NON-COMMUTATIVE BIPARTITE GRAPH}

As non-commutative bipartite graphs play an important role in zero-error classical communication, we will investigate the relationship between zero-error quantum capacity and noncommutative bipartite graph in this section. To be specific, we will prove that zero-error quantum capacities assisted with NS, PPTp or NS $\cap$ PPTp codes also depend only on the noncommutative bipartite graph of a quantum channel.

Let $P_{A B}$ denote the projection onto the support of the Choi-Jamiołkowski matrix of $\mathcal{N}$, which means that $P_{A B}$ is completely determined by $K(\mathcal{N})$. We also define the following SDP which only depends on $K$,

$$
\begin{gathered}
D^{\Omega}(K, k)=\max \operatorname{Tr} P_{A B}\left(W_{A B}-\rho_{A} \otimes \mathbb{1}_{B}\right) \\
\text { s.t. } 0 \leq W_{A B} \leq \rho_{A} \otimes \mathbb{1}_{B}, \operatorname{Tr} \rho_{A}=1, \\
\text { PPTp: }-\frac{\rho_{A} \otimes \mathbb{1}_{B}}{k} \leq W_{A B}^{T_{B}} \leq \frac{\rho_{A} \otimes \mathbb{1}_{B}}{k}, \\
\text { NS: } \operatorname{Tr}_{A} W_{A B}=\frac{1}{k^{2}} \mathbb{1}_{B} .
\end{gathered}
$$

Theorem 1 For a quantum channel $\mathcal{N}$ with non-commutative bipartite graph $K, F^{\Omega}(\mathcal{N}, k)=1$ if and only if $D^{\Omega}(K, k)=$ 0 . Furthermore, $Q_{0}^{\Omega,(1)}(\mathcal{N})=Q_{0}^{\Omega,(1)}(K)=\left\lfloor\kappa^{\Omega}(K)\right\rfloor$, where $\kappa^{\Omega}(K)=\max \left\{k: D^{\Omega}(K, k)=0, k \geq 0\right\}$.

Proof Firstly, noting that $\operatorname{Tr}\left(\rho_{A} \otimes \mathbb{1}_{B}\right) J_{A B}=\operatorname{Tr}_{A} \operatorname{Tr}_{B}\left[\left(\rho_{A} \otimes\right.\right.$ $\left.\left.\mathbb{1}_{B}\right) J_{A B}\right]=\operatorname{Tr} \rho_{A}=1$, we have that

$$
\begin{gathered}
F^{\Omega}(\mathcal{N}, k)-1=\max \operatorname{Tr} J_{A B}\left(W_{A B}-\rho_{A} \otimes \mathbb{1}_{B}\right) \\
\text { s.t. } 0 \leq W_{A B} \leq \rho_{A} \otimes \mathbb{1}_{B}, \operatorname{Tr} \rho_{A}=1, \\
\text { PPTp: }-\frac{\rho_{A} \otimes \mathbb{1}_{B}}{k} \leq W_{A B}^{T_{B}} \leq \frac{\rho_{A} \otimes \mathbb{1}_{B}}{k}, \\
\text { NS: } \operatorname{Tr}_{A} W_{A B}=\frac{1}{k^{2}} \mathbb{1}_{B} .
\end{gathered}
$$


It is evident that $F^{\Omega}(\mathcal{N}, k)-1=0$ if and only if $\operatorname{Tr} J_{A B}\left(W_{A B}-\rho_{A} \otimes \mathbb{1}_{B}\right)=0$. Noting that $W_{A B}-\rho_{A} \otimes \mathbb{1}_{B} \leq$ 0 , then $\operatorname{Tr} J_{A B}\left(W_{A B}-\rho_{A} \otimes \mathbb{1}_{B}\right)=0$ is equivalent to $\operatorname{Tr} P_{A B}\left(W_{A B}-\rho_{A} \otimes \mathbb{1}_{B}\right)=0$. Therefore, $F^{\Omega}(\mathcal{N}, k)=1$ if and only if $D^{\Omega}(K, k)=0$. Consequently, zero-error quantum capacity assisted with $\Omega$ codes also depends only on the noncommutative bipartite graph.

Theorem 2 The one-shot NS codes assisted quantum zeroerror capacity of a non-commutative bipartite graph $K$ is given by the interger part of $\kappa^{N S}(K)=\sqrt{\Upsilon(K)}$, where $\Upsilon(K)$ is the NS assisted zero-error classical capacity introduced in [21].

Proof We can first simplify $\kappa^{N S}(K)$ to

$$
\begin{aligned}
& \kappa^{N S}(K)=\max k \text { s.t. } \quad 0 \leq k^{2} W_{A B} \leq k^{2} \rho_{A} \otimes \mathbb{1}_{B}, \\
& \operatorname{Tr}_{A} k^{2} W_{A B}=\mathbb{1}_{B}, \\
& \operatorname{Tr} P_{A B}\left(k^{2} \rho_{A} \otimes \mathbb{1}_{B}-k^{2} W_{A B}\right)=0 .
\end{aligned}
$$

Then suppose that $U_{A B}=k^{2} W_{A B}$ and $k^{2} \rho_{A}=S_{A}$, therefore

$$
\begin{aligned}
& \kappa^{N S}(K)=\max \sqrt{\operatorname{Tr} S_{A}} \text { s.t. } 0 \leq U_{A B} \leq S_{A} \otimes \mathbb{1}_{B}, \\
& \operatorname{Tr}_{A} U_{A B}=\mathbb{1}_{B}, \\
& \operatorname{Tr} P_{A B}\left(S_{A} \otimes \mathbb{1}_{B}-U_{A B}\right)=0 .
\end{aligned}
$$

Hence, $\left[\kappa^{N S}(K)\right]^{2}=\Upsilon(K)$.

For a quantum channel $\mathcal{N}$ assisted PPTp codes, we can "borrow" a noiseless qudit channel $I_{d}$ whose zero-error quantum capacity is $d$, then we can use $\mathcal{N} \otimes I_{d}$ to transmit information. After the communication finishes we "pay back" the capacity of $I_{d}$. This kind of communication method was suggested in [27], [22], and was highly relevant to the notion of potential capacity recently studied by Winter and Yang [25]. Based on this model, we define the one-shot activated PPTp codes assisted zero-error quantum capacity (message number form) is

$$
\kappa_{a}^{P P T p}(\mathcal{N}):=\sup _{d \geq 2} \frac{\left\lfloor\kappa^{P P T p}\left(\mathcal{N} \otimes I_{d}\right)\right\rfloor}{d} .
$$

where $I_{d}$ is a noiseless qudit channel.

Proposition 3 For a quantum channel $\mathcal{N}$ and a qudit noiseless channel $I_{d}, F^{P P T p}\left(\mathcal{N} \otimes I_{d}, k d\right)=F^{P P T p}(\mathcal{N}, k)$. Consequently, $\kappa^{P P T p}\left(\mathcal{N} \otimes I_{d}\right)=d \kappa^{P P T p}(\mathcal{N})$.

Proof On one hand, it is easy to prove that for two quantum channel $\mathcal{N}_{1}$ and $\mathcal{N}_{2}$,

$$
F^{P P T p}\left(\mathcal{N}_{1}, k_{1}\right) F^{P P T p}\left(\mathcal{N}_{2}, k_{2}\right) \leq F^{P P T p}\left(\mathcal{N}_{1} \otimes \mathcal{N}_{2}, k_{1} k_{2}\right) \text {. }
$$

Thus, $F^{P P T p}(\mathcal{N}, k) \leq F^{P P T p}\left(\mathcal{N} \otimes I_{d}, k d\right)$.

On the other hand, suppose that $F^{P P T p}(\mathcal{N}, k)=u$, assume that the optimal solution to $\operatorname{SDP}$ (4) of $F^{P P T P}(\mathcal{N}, k)$ is $\left\{X_{1}, Y_{1}, V_{1}\right\}$. For a Hermitian operator $Z$, we define the positive part $Z_{+}$and the negative part $Z_{-}$to be the unique positive operators such that $Z=Z_{+}-Z_{-}$and $Z_{+} Z_{-}=0$. Let $X_{2}=0, Y_{2}=\left(\Phi_{d}^{T_{B^{\prime}}}\right)_{-}, V_{2}=\left(\Phi_{d}^{T_{B^{\prime}}}\right)_{+}$, where $\Phi_{d}$ is the unnormalized maximally entanglement $\left|\Phi_{d}\right\rangle\left\langle\Phi_{d}\right|$ with $\left|\Phi_{d}\right\rangle=\sum_{i=0}^{d-1}|i i\rangle$. Then, $\left\{X_{2}, Y_{2}, V_{2}\right\}$ is a feasible solution to SDP (4) of $F^{P P T P}\left(I_{d}, d\right)$. Furthermore, noting that $Y_{2}+V_{2}=$ $\left(\Phi_{d}^{T_{B}^{\prime}}\right)_{-}+\left(\Phi_{d}^{T_{B}^{\prime}}\right)_{+}=\mathbb{1}_{B B^{\prime}}$, we can assume that $X=X_{1} \otimes \Phi_{d}$, $Y-V=-\left(Y_{1}-V_{1}\right) \otimes\left(Y_{2}-V_{2}\right)=\left(Y_{1}-V_{1}\right) \otimes \Phi_{d}^{T_{B^{\prime}}}$ and $Y+V=\left(Y_{1}+V_{1}\right) \otimes\left(Y_{2}+V_{2}\right)=\left(Y_{1}+V_{1}\right) \otimes \mathbb{1}_{B B^{\prime}}$. Then it is easy to show that $\{u, X, Y, V\}$ is a feasible solution to the dual SDP of $F^{P P T p}\left(\mathcal{N} \otimes I_{d}, k d\right)$.

Hence, $F^{P P T p}\left(\mathcal{N} \otimes I_{d}, k d\right)=u=F^{P P T p}(\mathcal{N}, k)$.

Proposition 4 For a channel $\mathcal{N}, \kappa_{a}^{P P T p}(\mathcal{N})=\kappa^{P P T p}(\mathcal{N})$. Furthermore, $Q_{0, a}^{P P T p}(\mathcal{N})=Q_{0}^{P P T p}(\mathcal{N})$. Then,

$$
Q_{0}^{P P T p}\left(\mathcal{N} \otimes I_{d}\right)=Q_{0}^{P P T p}(\mathcal{N})+\log d .
$$

Proof Let us first consider the case that $\kappa^{P P T p}(\mathcal{N})$ is a rational number. W.l.o.g, we assume that $\kappa^{P P T P}(\mathcal{N})=\frac{t}{m}$, where $t$ and $m$ are positive integers. On one hand,

$$
\kappa_{a}^{P P T p}(\mathcal{N}) \geq\left\lfloor\kappa^{P P T p}(\mathcal{N}) \kappa^{P P T p}\left(I_{m}\right)\right\rfloor / m=\frac{t}{m} .
$$

On the other hand, by Proposition 3, we have

$$
\kappa_{a}^{P P T p}(\mathcal{N}) \leq \sup _{d \geq 1}\left[\kappa^{P P T p}\left(\mathcal{N} \otimes I_{d}\right) / d\right]=\kappa^{P P T p}(\mathcal{N}) .
$$

Hence, $\kappa_{a}^{P P T p}(\mathcal{N})=\kappa^{P P T p}(\mathcal{N})$ and $Q_{0, a}^{P P T p}(\mathcal{N})=$ $Q_{0}^{P P T p}(\mathcal{N})$. Finally, the case of irrational numbers can be solved by taking limit and using continuity arguments.

Example The $d$-dimensional Werner-Holevo channel is defined as $\mathcal{W}_{d}(\rho)=\frac{1}{d-1}\left(\mathbb{1}_{B} \operatorname{Tr} \rho-\rho^{T}\right) . \mathcal{W}_{d}$ is anti-degradable and hence has no quantum capacity. However, the asymptotic quantum capacity and the zero-error quantum capacity of PPTpreserving codes over $\mathcal{W}_{3}$ are both $\log \frac{d+2}{d}$ [3]. For this $\mathcal{W}_{d}$,

$$
Q_{0}^{P P T p}\left(\mathcal{W}_{d}\right)=\log \kappa_{a}^{P P T p}\left(\mathcal{W}_{d}\right)=\log \frac{d+2}{d} .
$$

We will first show a feasible solution $\left\{\rho_{A}, V_{A B}\right\}$ of $F^{P P T p}\left(\mathcal{W}_{d}, \frac{d+2}{d}\right)=1$. Let $\rho_{A}=\frac{1}{d} \mathbb{1}_{A}$ and $V_{A B}=$ $\left(\frac{1}{d+2} \mathbb{1}_{A B}-\frac{2}{d(d+2)} \Phi_{d}\right)^{T_{B}}$, where $\Phi_{d}$ is the unnormalized maximally entanglement $\left|\Phi_{d}\right\rangle\left\langle\Phi_{d}\right|$ with $\left|\Phi_{d}\right\rangle=\sum_{i=0}^{d-1}|i i\rangle$. It is easy to check that $\left\{\rho_{A}, V_{A B}\right\}$ is a feasible solution such that $F^{P P T p}\left(\mathcal{W}_{d}, \frac{d+2}{d}\right)=1$, which means that $\kappa^{P P T p}\left(\mathcal{W}_{d}\right) \geq$ $\frac{d+2}{d}$. Thus, $\log \kappa_{a}^{P P T p}\left(\mathcal{W}_{d}\right)=\log \frac{d+2}{d}=Q_{0}^{P P T p}\left(\mathcal{W}_{d}\right)$.

\section{A GENERAL UPPER BOUND OF QUANTUM CAPACITY}

Since computing the quantum capacity of a quantum channel is very difficult, we will introduce an SDP upper bound to evaluate the quantum capacity of any channel. Semidefinite programming (SDP) problems [28] can be solved by polynomial time algorithms [29]. The CVX software [30] allows one to solve SDPs efficiently.

To be specific, we define $Q_{\Gamma}(\mathcal{N})=\log \Gamma(\mathcal{N})$ and

$$
\begin{aligned}
& \Gamma(\mathcal{N})=\max \operatorname{Tr} J_{A B} R_{A B} \\
& \text { s.t. } \quad R_{A B}, \rho_{A} \geq 0, \operatorname{Tr} \rho_{A}=1, \\
& \quad-\rho_{A} \otimes \mathbb{1}_{B} \leq R_{A B}^{T_{B}} \leq \rho_{A} \otimes \mathbb{1}_{B} .
\end{aligned}
$$


The dual SDP is given by

$$
\begin{aligned}
\Gamma(\mathcal{N})= & \min \mu \\
\text { s.t. } & Y_{A B}, V_{A B} \geq 0,\left(V_{A B}-Y_{A B}\right)^{T_{B}} \geq J_{A B}, \\
& \operatorname{Tr}_{B}\left(V_{A B}+Y_{A B}\right) \leq \mu \mathbb{1}_{A} .
\end{aligned}
$$

By strong duality, the values of both the primal and the dual SDP coincide. This quantity also relates to Rains bound [19] and the improved SDP bound of distillable entanglement [31]. $Q_{\Gamma}$ has some remarkable properties. For example, it is additive: $Q_{\Gamma}(\mathcal{N} \otimes \mathcal{M})=Q_{\Gamma}(\mathcal{M})+Q_{\Gamma}(\mathcal{N})$ for different quantum channels $\mathcal{N}$ and $\mathcal{M}$. This can be proved by utilizing semi-definite programming duality.

Theorem 5 For quantum channels $\mathcal{M}$ and $\mathcal{N}, Q^{P P T p}(\mathcal{N})+$ $Q^{P P T p}(\mathcal{M}) \leq Q^{P P T p}(\mathcal{M} \otimes \mathcal{N}) \leq Q^{P P T p}(\mathcal{M})+Q_{\Gamma}(\mathcal{N})$.

Consequently,

$$
\begin{aligned}
Q(\mathcal{N}) & \leq Q^{F C A}(\mathcal{N}) \leq Q^{F H A}(\mathcal{N}) \\
& \leq Q^{P P T p}(\mathcal{N}) \leq Q_{p}^{P P T p}(\mathcal{N}) \leq Q_{\Gamma}(\mathcal{N}),
\end{aligned}
$$

where $\boldsymbol{F C A}, \boldsymbol{F H A}$ represent for forward-classical-assisted codes and forward-Horodecki-assisted codes, respectively.

Proof Firstly, from SDP (3), it is easy to see that $Q^{P P T p}(\mathcal{N})+Q^{P P T p}(\mathcal{M}) \leq Q^{P P T p}(\mathcal{M} \otimes \mathcal{N})$.

Secondly, assume that $Q^{P P T p}(\mathcal{M} \otimes \mathcal{N})=q$, then

$$
\lim _{n \rightarrow \infty} F^{P P T p}\left((\mathcal{N} \otimes \mathcal{M})^{\otimes n},\left\lfloor 2^{q n}\right\rfloor\right)=1 .
$$

Let $\Gamma(\mathcal{N})=t$, from Lemma 6 below, we have that

$$
\begin{aligned}
1 & \geq \lim _{n \rightarrow \infty} F^{P P T p}\left(\mathcal{M}^{\otimes n}, \frac{\left\lfloor 2^{q n}\right\rfloor}{t^{n}}\right) \\
& \geq \lim _{n \rightarrow \infty} F^{P P T p}\left((\mathcal{N} \otimes \mathcal{M})^{\otimes n},\left\lfloor 2^{q n}\right\rfloor\right)=1 .
\end{aligned}
$$

Let $Q^{P P T p}(\mathcal{M})=r$, then from the definition,

$$
\left\lfloor 2^{r n}\right\rfloor \geq \frac{\left\lfloor 2^{q n}\right\rfloor}{t^{n}}, n \rightarrow \infty .
$$

Then, it is easy to see that $t 2^{r} \geq\left(2^{q n}-1\right)^{1 / n}(n \rightarrow \infty)$, which means that $\log t+r \geq q$. Hence, $Q^{P P T p}(\mathcal{M} \otimes$ $\mathcal{N}) \leq Q^{P P T p}(\mathcal{M})+Q_{\Gamma}(\mathcal{N})$. Then we immediately have that $\bar{Q}^{P P T p}(\mathcal{N}) \leq Q_{p}^{P P T p}(\mathcal{N})=\sup _{\mathcal{M}}\left[Q^{P P T p}(\mathcal{M} \otimes \mathcal{N})-\right.$ $\left.Q^{P P T p}(\mathcal{M})\right] \leq Q_{\Gamma}(\mathcal{N})$.

Lemma 6 For quantum channels $\mathcal{N}_{1}$ and $\mathcal{N}_{2}$, we have that

$$
\begin{aligned}
& F^{P P T p}\left(\mathcal{N}_{1}, k\right) F^{P P T p}\left(\mathcal{N}_{2}, \Gamma\left(\mathcal{N}_{2}\right)\right) \\
\leq & F^{P P T p}\left(\mathcal{N}_{1} \otimes \mathcal{N}_{2}, k \Gamma\left(\mathcal{N}_{2}\right)\right) \leq F^{P P T p}\left(\mathcal{N}_{1}, k\right) .
\end{aligned}
$$

Proof It is easy to prove the first inequality. For the latter inequality, assume that the optimal solutions to dual SDPs of $F^{P P T p}\left(\mathcal{N}_{1}, k\right)$ and $\Gamma\left(\mathcal{N}_{2}\right)$ are $\left\{u_{1}, X_{1}, Y_{1}, V_{1}\right\}$ and $\left\{u_{2}, Y_{2}, V_{2}\right\}$, respectively. Let $X=X_{1} \otimes J_{2}, V-Y=$ $\left(V_{1}-Y_{1}\right) \otimes\left(V_{2}-Y_{2}\right), Y+V=\left(Y_{1}+V_{1}\right) \otimes\left(Y_{2}+V_{2}\right)$, then the idea is to prove that $\left\{u_{1}, X, Y, V\right\}$ is a feasible solution to dual SDP of $F^{P P T p}\left(\mathcal{N}_{1} \otimes \mathcal{N}_{2}, k \Gamma(\mathcal{N})\right)$, which means that $F^{P P T p}\left(\mathcal{N}_{1} \otimes \mathcal{N}_{2}, k \Gamma(\mathcal{N})\right) \leq F^{P P T p}\left(\mathcal{N}_{1}, k\right)$.
Corollary 7 For any two quantum channels $\mathcal{N}$ and $\mathcal{M}$, we have that $Q^{P P T p}(\mathcal{N} \otimes \mathcal{M}) \leq Q_{\Gamma}(\mathcal{N})+Q_{\Gamma}(\mathcal{M})$.

Remark In [26], the super-activation of quantum capacity says that two zero-capacity channels $(50 \%$ erasure channel $\mathcal{N}_{e}^{0.5}$ and a Horodecki channel $\left.\mathcal{N}_{H}\right)$ can have a nonzero capacity when used together, i.e. $Q\left(\mathcal{N}_{e}^{0.5} \otimes \mathcal{N}_{H}\right)>0.01$. Here, applying this corollary, we can evaluate the super-activation: $Q\left(\mathcal{N}_{e}^{0.5} \otimes \mathcal{N}_{H}\right) \leq Q_{\Gamma}\left(\mathcal{N}_{e}\right)+Q_{\Gamma}\left(\mathcal{N}_{H}\right)=Q_{\Gamma}\left(\mathcal{N}_{e}^{0.5}\right) \approx 1.123$

\section{COMPARISON WITH OTHER BOUNDS}

In [4], Holevo and Werner gave a general upper bound of quantum capacity for channel $\mathcal{N}$ with Choi-Jamiołkowski matrix $J_{\mathcal{N}}$ :

$$
Q(\mathcal{N}) \leq Q_{\Theta}(\mathcal{N})=\log \left\|J_{\mathcal{N}}^{T_{B}}\right\|_{c b}
$$

Here $\|\cdot\|_{c b}$ is the completely bounded trace norm, which is known to be efficiently computable by semidefinite program$\operatorname{ming}[32]$.

Theorem 8 For a quantum channel $\mathcal{N}$,

$$
Q(\mathcal{N}) \leq Q_{\Gamma}(\mathcal{N}) \leq Q_{\Theta}(\mathcal{N}),
$$

and both inequalities can be strict.

Proof Assume that the optimal solution of $\Gamma(\mathcal{N})$ is $\left\{R_{A B}, \rho_{A}\right\}$, then $\Gamma(\mathcal{N})=\operatorname{Tr} J_{\mathcal{N}} R_{A B}=\operatorname{Tr} J_{\mathcal{N}}^{T_{B}} R_{A B}^{T_{B}}$.

From Theorem 6 in [32],

$$
\begin{array}{r}
\left\|J_{\mathcal{N}}^{T_{B}}\right\|_{c b}=\max \frac{1}{2} \operatorname{Tr}\left(J_{\mathcal{N}}^{T_{B}} X\right)+\frac{1}{2} \operatorname{Tr}\left(J_{\mathcal{N}}^{T_{B}} X^{\dagger}\right) \\
\text { s.t. }\left(\begin{array}{cc}
\rho_{0} \otimes \mathbb{1} & X \\
X^{\dagger} & \rho_{1} \otimes \mathbb{1}
\end{array}\right) \geq 0 .
\end{array}
$$

Let us add two constraints $\rho_{0}=\rho_{1}=\rho_{A}$ and $X=X^{\dagger}$, then

$$
\left\|J_{\mathcal{N}}^{T_{B}}\right\|_{c b} \geq \max \operatorname{Tr}\left(J_{\mathcal{N}}^{T_{B}} X\right) \text { s.t. }\left(\begin{array}{cc}
\rho_{A} \otimes \mathbb{1} & X \\
X & \rho_{A} \otimes \mathbb{1}
\end{array}\right) \geq 0 .
$$

Noting that $-\rho_{A} \otimes \mathbb{1} \leq R_{A B}^{T_{B}} \leq \rho_{A} \otimes \mathbb{1}$, then

$$
\begin{aligned}
& \left(\begin{array}{cc}
\rho_{A} \otimes \mathbb{1} & R_{A B}^{T_{B}} \\
R_{A B}^{T_{B}} & \rho_{A} \otimes \mathbb{1}
\end{array}\right) \\
= & \frac{1}{2}\left(\begin{array}{cc}
\rho_{A} \otimes \mathbb{1}+R_{A B}^{T_{B}} & \rho_{A} \otimes \mathbb{1}+R_{A B}^{T_{B}} \\
\rho_{A} \otimes \mathbb{1}+R_{A B}^{T_{B}} & \rho_{A} \otimes \mathbb{1}+R_{A B}^{T_{B}}
\end{array}\right) \\
+ & \frac{1}{2}\left(\begin{array}{cc}
\rho_{A} \otimes \mathbb{1}-R_{A B}^{T_{B}} & -\left(\rho_{A} \otimes \mathbb{1}-R_{A B}^{T_{B}}\right) \\
-\left(\rho_{A} \otimes \mathbb{1}-R_{A B}^{T_{B}}\right) & \rho_{A} \otimes \mathbb{1}-R_{A B}^{T_{B}}
\end{array}\right) \geq 0 .
\end{aligned}
$$

Therefore, $R_{A B}^{T_{B}}$ satisfies the constraint above, which means that $\left\|J_{\mathcal{N}}^{T_{B}}\right\|_{c b} \geq \operatorname{Tr}\left(J_{\mathcal{N}}^{T_{B}} R_{A B}^{T_{B}}\right)=\Gamma(\mathcal{N})$. We will further compare our semidefinite programming upper bound $Q_{\Gamma}(\mathcal{N})$ to $Q_{\Theta}(\mathcal{N})$ in Fig. 1 based on $\mathcal{N}_{r}=\sum_{i} E_{i} \cdot E_{i}^{\dagger}(0 \leq r \leq 0.5)$ with $E_{0}=|0\rangle\langle 0|+\sqrt{r}| 1\rangle\langle 1|$ and $E_{1}=\sqrt{1-r}|0\rangle\langle 1|+| 1\rangle\langle 2|$.

Comparing with the upper bound $Q_{A D}$ induced by $\epsilon$ degradable quantum channels [6], $Q_{\Gamma}$ is tighter when $\epsilon$ is not small. For example, for the class of channel $\mathcal{N}_{r}$, when $r<0.38, Q_{\Gamma}<\epsilon \log 2+\left(1+\frac{1}{2} \epsilon\right) h\left(\frac{\epsilon}{2+\epsilon}\right) \leq Q_{A D}$. 


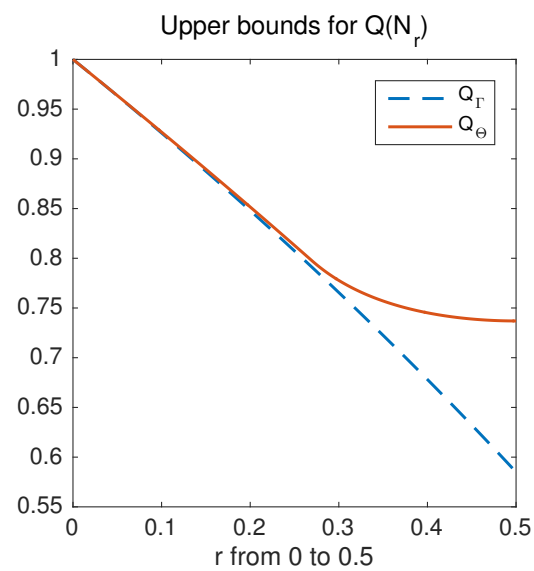

Fig. 1. This plot shows different upper bounds of $Q\left(\mathcal{N}_{r}\right)$. Dashed line depicts the upper bound $Q_{\Gamma}\left(\mathcal{N}_{r}\right)$ while solid line depicts $Q_{\Theta}\left(\mathcal{N}_{r}\right)$

\section{CONCLUSIONS}

We prove that the NS/PPTp/NS $\cap P P T p$ codes assisted zeroerror quantum capacity depends only on the non-commutative bipartite graph of the channel and the NS codes assisted zeroerror quantum capacity is given by the square root of the QSNC assisted zero-error classical capacity. We then introduce the activated PPTp codes assisted zero-error quantum capacity. Furthermore, we present a general SDP upper bound $Q_{\Gamma}$ of quantum capacity, which can be used to evaluate the quantum capacity of an arbitrary channel efficiently. $Q_{\Gamma}$ is always smaller than or equal to $Q_{\Theta}$ and can be strictly smaller than $Q_{\Theta}$ and $Q_{A D}$ for some channels. This upper bound is also additive and thus becomes an upper bound of the potential PPTp codes assisted capacity. $Q_{\Gamma}$ can also be used to bound the super-activation of quantum capacity.

One interesting open problem is to determine the asymptotic PPTp codes assisted zero-error quantum capacity $Q_{0}^{P P T p}(K)$. Also, it would be very interesting to combine the upper bound $Q_{\Gamma}$ with some entropy bounds such as the $Q_{s s}$ in [5].

\section{ACKNOWLEDGMENTS}

We were grateful to M. Tomamichel for helpful suggestions. This work was partly supported by the Australian Research Council (Grant No. DP120103776 and No. FT120100449) and the National Natural Science Foundation of China (Grant No. 61179030).

\section{REFERENCES}

[1] T. Cubitt, D. Elkouss, W. Matthews, M. Ozols, D. Pérez-García, and S. Strelchuk, "Unbounded number of channel uses may be required to detect quantum capacity," Nature communications, vol. 6, 2015.

[2] C. H. Bennett, I. Devetak, P. W. Shor, and J. A. Smolin, "Inequalities and separations among assisted capacities of quantum channels," Physical Review Letters, vol. 96, no. 15, p. 150502, 2006.

[3] D. Leung and W. Matthews, "On the Power of PPT-Preserving and NonSignalling Codes,” pp. 4486-4499, 2015.

[4] A. S. Holevo and R. F. Werner, "Evaluating capacities of bosonic Gaussian channels," Physical Review A, vol. 63, no. 3, p. 32312, 2001.

[5] G. Smith, J. Smolin, and A. Winter, "The quantum capacity with symmetric side channels," IEEE Transactions on Information Theory, vol. 54, no. 9, pp. 4208-4217, 2008 .
[6] D. Sutter, V. B. Scholz, and R. Renner, "Approximate Degradable Quantum Channels," arXiv:1412.0980, 2014.

[7] L. Gao, M. Junge, and N. LaRacuente, "Capacity Bounds via Operator Space Methods," arXiv:1509.07294, 2015.

[8] D. Bruß, D. P. DiVincenzo, A. Ekert, C. A. Fuchs, C. Macchiavello, and J. A. Smolin, "Optimal universal and state-dependent quantum cloning," Physical Review A, vol. 57, no. 4, p. 2368, 1998.

[9] N. J. Cerf, "Pauli cloning of a quantum bit," Physical Review Letters, vol. 84 , no. 19 , p. 4497,2000

[10] M. M. Wolf and D. Perez-Garcia, "Quantum capacities of channels with small environment," Physical Review A, vol. 75, no. 1, p. 12303, 2007.

[11] G. Smith and J. Smolin, "Additive extensions of a quantum channel," Proceedings of IEEE Information Theory Workshop (ITW) , pp. 368372, 2008.

[12] M. Tomamichel, M. M. Wilde, and A. Winter, "Strong converse rates for quantum communication," Proceedings of 2015 IEEE International Symposium on Information Theory (ISIT), pp. 2386-2390, 2015.

[13] S. Lloyd, "Capacity of the noisy quantum channel," Physical Review A, vol. 55, no. 3, p. 1613, 1997.

[14] P. W. Shor, "The quantum channel capacity and coherent information," in lecture notes, MSRI Workshop on Quantum Computation, 2002.

[15] I. Devetak, "The private classical capacity and quantum capacity of a quantum channel," IEEE Transactions on Information Theory, vol. 51, no. 1 , pp. 44-55, 2005.

[16] B. Schumacher and M. A. Nielsen, "Quantum data processing and error correction," Physical Review A, vol. 54, no. 4, p. 2629, 1996.

[17] H. Barnum, E. Knill, and M. A. Nielsen, "On quantum fidelities and channel capacities," IEEE Transactions on Information Theory, vol. 46, no. 4, pp. 1317-1329, 2000.

[18] H. Barnum, M. A. Nielsen, and B. Schumacher, "Information transmission through a noisy quantum channel," Physical Review A, vol. 57, no. 6, p. 4153,1998

[19] E. M. Rains, "A semidefinite program for distillable entanglement," IEEE Transactions on Information Theory, vol. 47, no. 7, p. 2921, 2001.

[20] T. S. Cubitt, D. Leung, W. Matthews, and A. Winter, "Zero-Error Channel Capacity and Simulation Assisted by Non-Local Correlations," IEEE Transactions on Information Theory, vol. 57, no. 8, pp. 55095523, 2011.

[21] R. Duan and A. Winter, "No-Signalling-Assisted Zero-Error Capacity of Quantum Channels and an Information Theoretic Interpretation of the Lovász Number," IEEE Transactions on Information Theory, vol. 62 , no. 2, pp. 891-914, 2016.

[22] R. Duan and X. Wang, "Activated zero-error classical capacity of quantum channels in the presence of quantum no-signalling correlations,' arXiv:1510.05437, 2015.

[23] R. Duan, S. Severini, and A. Winter, "Zero-error communication via quantum channels, noncommutative graphs, and a quantum Lovász number," IEEE Transactions on Information Theory, vol. 59, no. 2, pp 1164-1174, 2013.

[24] — " "On zero-error communication via quantum channels in the presence of noiseless feedback," arXiv:1502.02987, 2015.

[25] D. Yang and A. Winter, "Potential capacities of quantum channels," IEEE Transactions on Information Theory, vol. 62, no. 3, pp. 14151424, 2016

[26] G. Smith and J. Yard, "Quantum communication with zero-capacity channels," Science, vol. 321, no. 5897, pp. 1812-1815, 2008.

[27] A. Acín, R. Duan, A. B. Sainz, and A. Winter, "A new property of the Lovász number and duality relations between graph parameters," arXiv:1505.01265, 2015.

[28] L. Vandenberghe and S. Boyd, "Semidefinite programming," SIAM review, vol. 38, no. 1, pp. 49-95, 1996.

[29] L. G. Khachiyan, "Polynomial algorithms in linear programming," USSR Computational Mathematics and Mathematical Physics, vol. 20, no. 1, pp. 53-72, 1980 .

[30] M. Grant and S. Boyd, "CVX: Matlab software for disciplined convex programming," 2008. [Online]. Available: http://cvxr.com/cvx

[31] X. Wang and R. Duan, "An Improved Semidefinite Programming Upper Bound on Distillable Entanglement," arXiv:1601.07940, 2016.

[32] J. Watrous, "Simpler semidefinite programs for completely bounded norms," arXiv:1207.5726, 2012 